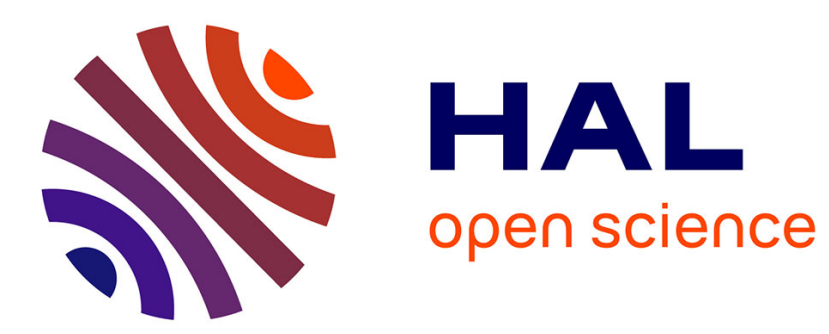

\title{
Signal Representation and Segmentation based on Multifractal Stationarity
}

\author{
Khalid Daoudi, Jacques Lévy Véhel
}

\section{To cite this version:}

Khalid Daoudi, Jacques Lévy Véhel. Signal Representation and Segmentation based on Multifractal Stationarity. Signal Processing, 2002, 82 (12), pp.2015-2024. 10.1016/S0165-1684(02)00198-6 . inria00100882

\section{HAL Id: inria-00100882 \\ https://hal.inria.fr/inria-00100882}

Submitted on 13 Nov 2020

HAL is a multi-disciplinary open access archive for the deposit and dissemination of scientific research documents, whether they are published or not. The documents may come from teaching and research institutions in France or abroad, or from public or private research centers.
L'archive ouverte pluridisciplinaire HAL, est destinée au dépôt et à la diffusion de documents scientifiques de niveau recherche, publiés ou non, émanant des établissements d'enseignement et de recherche français ou étrangers, des laboratoires publics ou privés. 


\title{
Signal Representation and Segmentation based on Multifractal Stationarity
}

\author{
Khalid Daoudi ${ }^{1}$ and Jacques Lévy Véhel $^{2}$ \\ 1: INRIA-LORIA \\ B.P. 101 - 54602 Villers les Nancy. France \\ 2: Projet Fractales, INRIA Rocquencourt \\ B.P. 105 - 78153 Le Chesnay. France \\ email: daoudi@loria.fr, Jacques.Levy-Vehel@inria.fr
}

\begin{abstract}
We present a new scheme for signal representation which is well suited for the study of multifractal features. In particular, our approach, which is based on the use of Weakly Self Affine functions, allows to segment a signal into parts which are "multifractally homogeneous". Furthermore, it opens the possibility of estimating non concave multifractal spectra, a valuable improvement for many practical applications such as Internet traffic modeling.
\end{abstract}

Keywords: Self-similar functions, weakly self-affine functions, multifractal formalism, multifractal signal analysis, multifractal stationarity, wavelet analysis.

\section{Introduction}

Among the numerous possibilities for signal representations, a subclass focuses on yielding a faithful reproduction of the fractal properties. Such representations may be parametric or model free. The main feature of parametric representations is that the signal at hand is supposed to be "fractal" in some sense, in fact in the sense defined by the model. The most well-known example here is IFS-based modeling [1], where one tries to find an IFS whose attractor is close to the given signal. In the model free approach, one does not make such an assumption, but rather aims at defining a representation which has the two features of being general enough to handle a wide variety of signals (i.e. non "fractal" ones) and of inheriting the fractal properties of the original data. Generalized iterated functions systems (GIFS) provide such a representation of signals [2]. GIFS are an extension of IFS where the contractions, their number and their support vary at each iteration of the attractor generation process. GIFS were designed to construct functions with prescribed "arbitrary" local Hölder regularity. Another example is the PIFS (partitioned IFS [1]), which allow to model any image with prescribed accuracy and are used in image compression. Let us give an example that explains what we mean by a representation that allows to keep track of the fractal properties of a signal. Assume we are given discrete data $X=\left(X_{1}, \ldots, X_{N}\right)$. We may rightfully represent this signal using a e.g. spline that will interpolate the $X_{i}$. Alternatively, it is possible to define a GIFS that 
will also interpolate the data. However, the properties of the two representations are vastly different: for instance, the box dimension in the first case is one, the Hölder exponent is infinite at each point and the multifractal spectrum is in consequence degenerated. On the other hand, the gradient is well defined along the curve. The situation is exactly inverse for the GIFS : in general, the box dimension will be strictly greater than one, the Hölder exponents will vary wildly in time resulting in a non trivial multifractal spectrum, while the derivative may not exist at any point. Of course, the question of which representation is better depends on a priori knowledge on the data, on the kind of properties one is interested in, and/or on which processing needs to be applied afterwards to the signal. For instance, it has been remarked that Internet traffic [3, 4] display fractal features ; if one is interested in detecting structural changes in traffic, or in segmenting a given log into homogeneous parts, it makes sense to base such a processing on features which are considered important for the application, such as long range dependence or Hölder regularity. In doing so, one might want to make as few assumptions as possible on the data. In such a case, a GIFS-based representation is adequate.

However, GIFS, whose attractors are dense in $L^{2}$, are in some sense too general. In particular, it is not possible to derive an analytical formula for the multifractal spectrum of a generic GIFS. It is our aim in this work to introduce a new representation of signals, based on Weakly Self Affine (WSA) functions, which are less general than GIFS but, contrarily to GIFS, allow exact computation of most fractal quantities of interest. WSA functions are versatile enough to model a wide variety of signals while having enough structure so that their multifractal spectrum and Hölder exponents can be computed in an analytical way. This new representation might be advantageous in many cases. For instance, in the particular case of speech signals, we will see how this new representation may be used for segmentation purposes.

WSA functions are introduced in section 2, along with their main (multi)fractal properties. Using wavelet decompositions, we present, in section 3, a technique to find a WSA function which approximates "at best" a given signal. This technique has the advantage of allowing efficient segmentation, thus leading to more accurate representations. We describe a method for this WSA-based segmentation of signals in section 4 . When a real signal has been modeled by such a lumping of WSA functions, it is an easy task to estimate its multifractal spectrum. Indeed, each part is multifractally stationary in the sense that it is well modeled by a single multifractal object. We explain this in details in section 5 . An important feature then is that the spectrum so estimated does not have to be concave. Thus WSA modeling allows to obtain robust non concave spectra, in an analytical way. This appears to be new in the literature. In Section 6, we present some applications on real data (voice signals and Internet traffic).

\section{Weakly self-affine functions and multifractal formalism}

WSA functions are defined as a generalization of self-similar functions, in the sense of [5], where the similarity ratios are allowed to vary at each scale. Formally:

Definition 1 A function $f:[0,1] \rightarrow \mathbb{R}$ is called weakly self-affine iff:

i) There exists an open set $\Omega \subset[0,1]$ and $d(d \geq 2)$ contractive similitudes $S_{0}, \ldots, S_{d-1}$ all of ratio $\frac{1}{d}$, such that: 
- $S_{i}(\Omega) \subset \Omega \forall i \in\{0, \ldots, d-1\}$

- $S_{i}(\Omega) \cap S_{j}(\Omega)=\emptyset$ if $i \neq j$

ii) There exists $d$ positive sequences $\left(\lambda_{0}^{j}\right)_{j \in \mathbb{N}^{*}}, \ldots,\left(\lambda_{d-1}^{j}\right)_{j \in \mathbb{N}^{*}}$ satisfying $0<\lambda_{i}^{j}<1$ for every $i \in\{0, \ldots, d-1\}$ and $j \in \mathbb{N}^{*}$, and there exists a compactly supported continuous function $g$ such that $f$ verifies:

$$
f(x)=g(x)+\sum_{n=1}^{\infty} \sum_{\left(i_{1}, \ldots, i_{n}\right) \in\{0, \ldots, d-1\}^{n}}\left(\prod_{j=1}^{n} \epsilon_{\sum_{p=1}^{j} i_{p^{2}}{ }^{j-p}} \lambda_{i_{j}}^{j}\right) g\left(S_{i_{n}}^{-1} \circ \ldots \circ S_{i_{1}}^{-1}(x)\right)
$$

where, for each $j \geq 1$ and $k \in\left\{0, \ldots, d^{j}-1\right\}$, we have $: \epsilon_{k}^{j}= \pm 1$.

If there exist $d$ reals $\lambda_{0}, \ldots, \lambda_{d-1}$ such that

$$
\epsilon_{k}^{j} \lambda_{i}^{j}=\lambda_{i}, \quad \forall i \in\{0, \ldots, d-1\}, \forall j \geq 1 \text { and } \forall k \in\left\{0, \ldots, d^{j}-1\right\},
$$

then one recovers the classical self-similar functions, in the sense of [5]. The (weak) selfaffinity of $f$ is apparent when one realizes that Definition 1 implies that $f$ can be obtained as the limit of the sequence $\left(f_{j}\right)_{j \in \mathbb{N}}$, where $f_{0}(x)=g(x)$ and, for $j \geq 1, f_{j}$ is recursively computed as follows:

$$
f_{j}(x)=\sum_{i=0}^{d-1} \epsilon_{i}^{j} \lambda_{i}^{j} f_{j-1}\left(S_{i}^{-1}(x)\right)+g(x) .
$$

The following theorem, proved in [6], allows to compute the multifractal spectrum $d(\alpha)$ of WSA functions. The corresponding theorem for classical self-similar functions can be found in [5]. Define, for every integer $j \geq 1$, the $d$-tuple $\left(u_{0}^{j}, \ldots, u_{d-1}^{j}\right)$ by:

$$
\left(u_{0}^{j}, \ldots, u_{d-1}^{j}\right)=\left(\lambda_{i_{0}}^{j}, \ldots, \lambda_{i_{d-1}}^{j}\right),
$$

where $\left(i_{0}, \ldots, i_{d-1}\right)$ is the permutation of $(0, \ldots, d-1)$ which yields:

$$
\lambda_{i_{0}}^{j} \leq \ldots \leq \lambda_{i_{d-1}}^{j} .
$$

In other words, for each $j,\left(u_{0}^{j}, \ldots, u_{d-1}^{j}\right)$ is the $d$-tuple $\left(\lambda_{0}^{j}, \ldots, \lambda_{d-1}^{j}\right)$ rearranged in the increasing order. Then,

Theorem 1 Suppose that there exists two reals $a>0$ and $b>0$ such that, for every $i \in\{0, \ldots, d-1\}$ and $j \geq 1$ we have:

$$
0<a \leq u_{i}^{j} \leq b<1
$$

Suppose also that:

$$
p\left(x_{0}, \ldots, x_{d-1}\right)=\lim _{n} \frac{\operatorname{card}\left\{j \in\{1, \ldots, n\}: u_{i}^{j} \leq x_{i} \forall i=0, \ldots, d-1\right\}}{n}
$$

exists for every $\left(x_{0}, \ldots, x_{d-1}\right) \in[a ; b]^{d}$. Suppose finally that $g$ is uniformly more regular than $f$. Let $d(\alpha)$ denote the Hausdorff multifractal spectrum of $f$, i.e. $d(\alpha)=\operatorname{dim}_{H}\{x$ : $\alpha(x)=\alpha\}$, where $\alpha(x)$ is the Hölder exponent of $f$ at $x$. Then : 
- $d(\alpha)=-\infty$ if $\alpha \notin\left[\alpha_{\min } ; \alpha_{\max }\right]$ where

$$
\left\{\begin{array}{c}
\alpha_{\min }=\lim _{n}-\frac{\log _{d}\left(u_{d-1}^{1}\right)+\ldots+\log _{d}\left(u_{d-1}^{n}\right)}{n} \\
\alpha_{\max }=\lim _{n}-\frac{\log _{d}\left(u_{0}^{1}\right)+\ldots+\log _{d}\left(u_{0}^{n}\right)}{n}
\end{array}\right.
$$

- if $\alpha \in\left[\alpha_{\min } ; \alpha_{\max }\right]$, then

$$
d(\alpha)=\inf _{q \in \mathbb{R}}(q \alpha-\tau(q))
$$

where

$$
\tau(q)=\liminf _{n \rightarrow \infty}-\frac{\sum_{j=1}^{n} \log _{d}\left(\left(\lambda_{0}^{j}\right)^{q}+\ldots+\left(\lambda_{d-1}^{j}\right)^{q}\right)}{n} .
$$

This theorem shows that a multifractal formalism is valid for WSA functions. As a consequence, the Hausdorff, Large Deviation and Legendre multifractal spectra coincide (see [3] for definitions), are concave, and may be computed easily. Let us finally recall that it is also possible to write an analytical formula for $\alpha(x)$ at all $x$. This follows easily by specializing the formula given in [2] for GIFS. Such a formula will not be needed in this work.

\section{Signal representation with WSA functions}

As mentioned above, our approach for representing a given signal is based on approximating it by one or several WSA functions. In this section, we develop a practical technique for the $L^{2}$-approximation of a signal by one WSA function. In practice only discrete data are available, therefore in the rest of the paper, we suppose that we are given a signal $\left\{f(m), m=0, \ldots, 2^{J}-1\right\}$. In the continuous setting, a WSA function is represented by an infinite number of parameters (the $\lambda_{i}^{j}$ 's). In the discrete setting, it does not make sense to represent a finite signal by infinite number of parameters. Thus, we make the assumption that WSA functions are also those for which the sequences $\left(\lambda_{i}^{j}\right)$ are finite, which makes finite the first summation in (1). We make also the assumption that theorem 1 remains true in this case.

Our problem is to find the parameters $\left(d, g,\left(S_{i}\right)_{i},\left(\epsilon_{k}^{j}\right)_{k, j},\left(\lambda_{i}^{j}\right)_{i, j}\right)$ of the WSA function that yields the best $L^{2}$-approximation of $f$. In its general form, this problem is hard to solve. However, it is possible to consider a simplified and less general version for which a solution can be found using an efficient algorithm based on a wavelet decomposition of $f$. We now proceed to describe this sub-optimal solution. Let $\phi$ be a scaling function, $\psi$ the corresponding wavelet and $w_{k}^{n}$ the wavelet coefficients of the signal $f$ :

$$
f(x)=a_{0} \phi(x)+\sum_{j=0}^{J-1} \sum_{0 \leq k<2^{j}} w_{k}^{j} \psi\left(2^{j} x-k\right) .
$$

Suppose that, for all $(k, j), w_{k}^{j} \neq 0$ and write for $j \geq 1$

$$
c_{k}^{j}=\left|\frac{w_{k}^{j}}{w_{\left[\frac{k}{2}\right]}^{j-1}}\right| \text {. }
$$


Set $S_{i}(x)=\frac{x+i}{2}$ for $i=0,1$. Then, a simple calculation yields:

$$
\begin{gathered}
f(x)=a_{0} \phi(x)+w_{0}^{0} \psi(x)+ \\
w_{0}^{0} \sum_{n=1}^{J-1} \sum_{\left(i_{1}, \ldots, i_{n}\right) \in\{0,1\}^{n}} \operatorname{sgn}\left(w_{0}^{0} w_{\sum_{p=1}^{n}{ }_{n}^{n} i_{p} 2^{n-p}}\right)\left(\prod_{j=1}^{n} c_{\sum_{p=1}^{j} i_{p} 2^{j-p}}^{j}\right) \psi\left(S_{i_{n}}^{-1} \circ \ldots \circ S_{i_{1}}^{-1}(x)\right),
\end{gathered}
$$

where $\operatorname{sgn}(x)$ denotes the sign of $x$. This last equality suggests the following sub-optimal choice for the parameters: if we add the constraint that $d=2$, we see that $\frac{f(x)-a_{0} \phi(x)}{w_{0}^{0}}$ takes a form reminiscent of (1) if we set $g=\psi, S_{i}(x)=\frac{x+i}{2}$ for $i=0,1$, and $\epsilon_{k}^{j}=\operatorname{sgn}\left(\frac{w_{k}^{j}}{w_{\left[\frac{k}{2}\right]}^{j-1}}\right)$. We thus fix from now on the values of $d, S_{0}$ and $S_{1}$ as above, and try to find optimal $g$ and $\left(\lambda_{i}^{j}\right)$.

Let us assume for the moment that $g$ is known. Our problem is then to seek, for each $j$, two positive reals $\lambda_{0}^{j}$ and $\lambda_{1}^{j}$ such that when we replace all the $\left(c_{2 k}^{j}\right)$ (resp. the $\left.\left(c_{2 k+1}^{j}\right)\right)$ by $\lambda_{0}^{j}$ (resp. $\lambda_{1}^{j}$ ) in the equation above, we obtain the best $L^{2}$-approximation of the original signal $f$. In other words, for a given couple $(\phi, \psi)$, we wish to find, at each scale $j$, one number that "represents best" the wavelet coefficients ratios of $f$ with even $k$ index, and the same for coefficients with odd $k$ index. Using a gradient descent in the wavelet domain, we showed in [6] that the two sequences $\left(\lambda_{0}^{n}\right)_{n \geq 1}$ and $\left(\lambda_{1}^{n}\right)_{n \geq 1}$, that solve the so simplified inverse problem are given by:

$$
\lambda_{i}^{1}=c_{i}^{1} \text { and } \lambda_{i}^{n}=\sum_{0 \leq k<2^{n-1}} P_{k}^{n} c_{2 k+i}^{n}
$$

for all $n>1$, where, for all $k \in\left\{0, \ldots, 2^{n-1}-1\right\}$,

$$
P_{k}^{n}=\frac{c_{k}^{n-1} \prod_{j=1}^{n-1}\left(\lambda_{i_{j}(k)}^{j}\right)^{n-j}}{\prod_{j=1}^{n-1}\left(\left|\lambda_{0}^{j}\right|^{2}+\left|\lambda_{1}^{j}\right|^{2}\right)}
$$

where $\left(i_{1}(k), \ldots, i_{n-1}(k)\right)$ is the unique sequence of $\{0,1\}^{n-1}$ such that $k=\sum_{j=1}^{n-1} i_{j}(k) 2^{n-j-1}$. In addition, for every $n>1$, we have : $\sum_{0 \leq k<2^{n-1}} P_{k}^{n}=1$.

Unfortunately, the above procedure does not lead to good representations in practice. The reasons may be analyzed as follows. Recall that each $c_{k}^{j}$ is defined as a ratio of two wavelet coefficients. While we assume that all the $w_{k}^{j}$ are non zero, arbitrarily small values will occur in most real situations. This will in general result in both huge and very small values for $c_{k}^{j}$. Obviously, such a wide range of values is not favorable to a modeling like the one we have in mind, where all the coefficients $\left\{c_{k}^{j}, k=0, \ldots, 2^{j}-1\right\}$ are replaced by two values: we need some control on the dispersion of the $\left(c_{k}^{j}\right)$. Now remember that we are interested in representing signals which are irregular (otherwise a fractal approach does not make sense). For such signals, there is energy at most scales and most positions, so that in turn most $c_{k}^{j}$ are in an intermediate range. In addition, from a fractal point of view, we are not interested in large $c_{k}^{j}$ because they do not contribute to the regularity 
of $f$ (see [2] for more details). Also, if we assume that $f$ is nowhere differentiable, the Hölder exponent at each point is smaller than one, and "many" $c_{k}^{j}$, including the ones that control the multifractal properties of $f$, will belong to $\left[\frac{1}{2}, 1\right]$. It thus seems reasonable to discard in our modeling the "large" $c_{k}^{j}$ and to take into account only the ones which are smaller than 1 . More precisely, we keep unchanged the $\left(c_{k}^{j}\right)$ whose value is greater than 1 and compute the $\left(\lambda_{i}^{j}\right)$ that give the best $L^{2}$-approximation to the signal considering only the remaining $\left(c_{k}^{j}\right)$. Of course, for such a strategy to make sense, it is necessary that the cardinal of $\left\{c_{k}^{j}: c_{k}^{j} \geq 1\right\}$ be negligible w.r.t the cardinal of $\left\{c_{k}^{j}: c_{k}^{j}<1\right\}$. This will depend on the nature of the signal and on the exact shape of $g=\psi$. These constraints lead to the following criterion for a sub-optimal choice of the analyzing wavelet ; $g=\psi$ is chosen so that:

- (C1) $\left\{w_{k}^{j}=0\right\}=\emptyset$

- (C2) the cardinality of $\left\{c_{k}^{j}: c_{k}^{j}<1\right\}$ is maximal

Because of boundary artifacts, another requirement is that we stop the wavelet expansion at a certain scale $j_{0}>0$ as is usual in wavelet processing. As a consequence, the $\left(c_{k}^{j}\right)$ are defined only for $j>j_{0}$. To sum up, if the signal $f$ is written

$$
f(x)=\sum_{0 \leq k<2^{j_{0}}} a_{k}^{j_{0}} \phi\left(2^{j_{0}} x-k\right)+\sum_{n=j_{0}}^{J-1} \sum_{0 \leq k<2^{n}} w_{k}^{n} \psi\left(2^{n} x-k\right),
$$

our problem is to find, for each $j>j_{0}$, two positive reals $\lambda_{0}^{j}$ and $\lambda_{1}^{j}$ such that when we replace all the $\left(c_{2 k}^{j}\right)$ verifying $c_{2 k}^{j}<1$ (resp. the $\left(c_{2 k+1}^{j}\right)$ verifying $c_{2 k+1}^{j}<1$ ) by $\lambda_{0}^{j}$ (resp. $\lambda_{1}^{j}$ ), we obtain the best $L^{2}$-approximation of the original signal $f$. The resulting approximating signal $\tilde{f}$ is then a WSA function defined by

$$
\begin{aligned}
& \tilde{f}(x)=\sum_{0 \leq k<2^{j_{0}}} a_{k}^{j_{0}} \phi\left(2^{j_{0}} x-k\right)+\sum_{0 \leq k<2^{j_{0}}} w_{k}^{j_{0}} \psi\left(2^{j_{0}} x-k\right)+ \\
& \sum_{n=j_{0}+1}^{J-1} \sum_{\left(i_{1}, \ldots, i_{n}\right) \in\{0,1\}^{n}}\left|w_{\sum_{p=1}^{j_{0}} i_{p} 2^{j_{0}-p}}\right| \operatorname{sgn}\left(w_{\sum_{p=1}^{n} i_{p} 2^{n-p}}^{n}\right)\left(\prod_{j=j_{0}+1}^{n} \tilde{c}_{\sum_{p=1}^{j} i_{p} 2^{j-p}}^{j}\right) \psi\left(S_{i_{n}}^{-1} \circ \ldots \circ S_{i_{1}}^{-1}(x)\right)
\end{aligned}
$$

where

$$
\tilde{c}_{\sum_{p=1}^{j} i_{p} 2^{j-p}}^{j}=\left\{\begin{array}{ll}
c_{\sum_{p=1}^{j} i_{p} 2^{j-p}}^{j} & \text { if } c_{\sum_{p=1}^{j} i_{p} 2^{j-p}}^{j} \geq 1 \\
\lambda_{i_{j}}^{j} & \text { otherwise }
\end{array} .\right.
$$

Note that, for $n>j_{0}$ and $k \in\left\{0, \ldots, 2^{n}-1\right\}$, the wavelet coefficient $\tilde{w}_{k}^{n}$ of $\tilde{f}$ is given by

$$
\left.\tilde{w}_{k}^{n}=\operatorname{sgn}\left(w_{k}^{n}\right) \mid w_{\left[\frac{k}{2^{n-j}}\right]}^{j_{0}}\right] \mid \prod_{j=j_{0}+1}^{n} \tilde{c}_{\left[\frac{k}{2^{n-j}}\right]}^{j} .
$$

Since we restrict to orthonormal wavelet transforms, which conserves energy, our goal is to find two positive sequences $\left(\lambda_{0}^{n}\right)_{n=j_{0}+1, \ldots, J-1}$ and $\left(\lambda_{1}^{n}\right)_{n=j_{0}+1, \ldots, J-1}$ which realize

$$
\operatorname{argmin} \sum_{n=j_{0}+1}^{J-1} \sum_{0 \leq k<2^{n}}\left|w_{k}^{n}-\tilde{w}_{k}^{n}\right|^{2} .
$$


Unfortunately, finding this global minimum is a difficult problem. We can however compute a local minimum by finding successively, for $n=\left\{j_{0}+1, \ldots, J-1\right\}$, the couple $\left(\lambda_{0}^{n}, \lambda_{1}^{n}\right)$ which realizes

$$
\operatorname{argmin} \sum_{0 \leq k<2^{n}}\left|w_{k}^{n}-\tilde{w}_{k}^{n}\right|^{2}
$$

The solution of $(P)$ is given in the following proposition (see the appendix for the proof).

Proposition 1 For $i=0,1$ and $n>j_{0}$, the $\left(\lambda_{i}^{n}\right)$ 's which solve $(P)$ are recursively given by

$$
\begin{aligned}
& \lambda_{i}^{j_{0}+1}=\frac{\sum_{\substack{0 \leq k<2^{j_{0}} \\
c_{2 k+i}^{j_{0}+1} \mid<1}}\left|w_{k}^{j_{0}}\right|^{2} c_{2 k+i}^{j_{0}+1}}{\sum_{\substack{0<k<2 \\
j j_{0} j_{0}+1}}\left|w_{k}^{j_{0}}\right|^{2}} \\
& \lambda_{i}^{n}=\frac{\sum_{\substack{0 \leq k<2^{n-1} \\
\left|c_{2 k+i}^{k}\right|<1}}\left\{\left|w_{\left[\frac{2 k+i}{\left.2^{n-j}\right]}\right]}^{j_{0}}\right|^{2} \prod_{j=j_{0}+1}^{n-1} \tilde{c}_{\left[\frac{2 k+i}{2^{n-j}}\right]}^{j} c_{\left[\frac{2 k+i}{2^{n-j}}\right]}^{j}\right\} c_{2 k+i}^{n}}{\sum_{\substack{0 \leq k<2^{n-1} \\
\left|c_{2 k+i}^{k}\right|<1}}\left|w_{\left[\frac{2 k+i}{2^{n-j}}\right]}^{j_{0}}\right|^{2}\left|\prod_{j=j_{0}+1}^{n-1} \tilde{c}_{\left[\frac{2 k+i}{2^{n-j}}\right]}^{j}\right|^{2}} \text { for } n=j_{0}+2, \ldots, J-1 .
\end{aligned}
$$

Formula (3), (4) and (5) completely define the WSA approximation of $f$.

Remark: It is of course possible to develop a similar algorithm for classical selfsimilar functions. However, this would constraint the search to a much smaller class than the one of WSA functions. This would lead in general to less accurate representations and, consequently, to less accurate multifractal spectra estimations. Moreover, since classical self-similar functions are a special instance of WSA functions, then our algorithm can also capture signals which are well represented by classical self-similar functions, namely the resulting $\lambda_{i}^{n}$ of equation (5) would be equal for each $n$.

\section{Signal segmentation with WSA functions}

In many situations, some signals are not well represented by a single WSA function. An obvious example is the lumping of two IFS : consider a signal $X$ on $[0,1]$ whose restrictions to $[0,1 / 2]$ and $[1 / 2,1]$ are attractors of two different IFS. Using a single WSA function to model $X$ will in general result in a large $L^{2}$ error, while two WSA functions would yield a perfect match (using the Schauder basis as the wavelet decomposition). It is thus desirable to design an automated procedure for segmenting a given signal into parts, each of which can be well represented by a WSA function. As in the previous section, an optimal algorithm for performing this kind of segmentation seems out of reach, and we consider in the following a greedy method that gives good results in practice.

Consider the dyadic tree of depth $J$ where, by convention, the root node is at level 0 and the leaf nodes are at level $J-1$ and where the nodes, at each level $j \in\{0, \ldots, J-1\}$, are labeled from left to right by $(l, j)$ with $l=0, \ldots, 2^{j}-1$. To each node $(l, j)$ such that 
$j>j_{0}$, we associate the coefficient $c_{l}^{j}$. The segmentation algorithm is based on the fact that the sub-tree descending from a node $(l, j)$ determines completely the restriction of $f$ to $I(l, j)=\left\{l 2^{J-j}, \ldots,(l+1) 2^{J-j}-1\right\}$. The idea is to define and measure the error associated to each node $(l, j)$ by the $L^{2}$ distance between the restriction of the original signal to $I(l, j)$ and the representation by a single WSA function of this restriction. In order to take into account the fact that errors at coarser scales are more important than at finer scales, we further weight this error measure (see below). Starting from the root node, which corresponds of course to a single WSA function for the whole signal, we split recursively the tree until, for each subtree, the error falls below some predefined threshold $\epsilon$. Each subtree thus determined is "well" represented by a single WSA function, and the union of the corresponding $I(l, j)$ defines the segmentation of $f$.

More precisely, to each node $(l, j)$ and each $n>j$, we associate the set of integers $I(l, j, n)=\left\{l 2^{n-j}, \ldots,(l+1) 2^{n-j}-1\right\}$ and we denote by $\lambda_{i}^{n}(l, j)$ a ratio of sums similar to the one in (5) but where the indices are determined by $\left(k \in I(l, j, n-1), c_{2 k+i}^{n}<1\right)$. Denote

$$
\begin{gathered}
e_{i}^{n}(l, j)=\left.\frac{1}{2^{n-j}} \sum_{\substack{k \in I(l, j, n-1) \\
c_{2 k+i}^{(n<1}}}\left|\lambda_{i}^{n}(l, j)-\right| c_{2 k+i}^{n}\right|^{2}, \\
e_{i}(l, j)=\sum_{n=j+1}^{J-1} e_{i}^{n}(l, j)
\end{gathered}
$$

and

$$
e(l, j)=\frac{e_{0}(l, j)+e_{1}(l, j)}{\sigma(j)}
$$

where $\sigma$ is a non-decreasing positive function. $e(l, j)$ is our error function and the function $\sigma$ is introduced to compensate for the fact that errors introduced at coarser scales are more important than at finer scales. We may now write our segmentation algorithm:

\section{Segmentation algorithm:}

Fix an $\epsilon>0$;

node $=$ root node; (this is the initialization)

function segmentation (node)

Begin

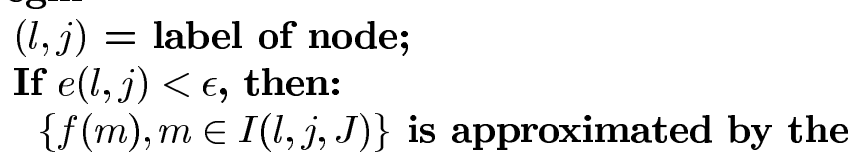

WSA function defined by $\left\{\lambda_{i}^{n}(l, j), n=j+1, \ldots, J-1, i=0,1\right\}$

else

segmentation (left child of node);

segmentation (right child of node);

\section{End}

This algorithm ends up segmenting $f$ in consecutive parts, each of which is well represented by a WSA function. In this sense, this method is a segmentation device of a new type: instead of cutting the original signal into parts which are homogeneous with respect to a classical criterion such as a local mean or even a fractional dimension, we 
use a criterion based on multifractal stationarity; indeed, each segment has a well defined multiplicative structure, with a multifractal spectrum given by theorem 1 , and the rule for splitting the signal follows from this requirement. As an application of this new segmentation scheme, we show in the next section how it allows to estimate non concave multifractal spectra.

Remark: As presented above, this algorithm suffers form the defect that cuts always occur at dyadic points. This implies a huge waste if the "true" segments are not well aligned with the dyadic grid. This is a common problem when one uses dyadic wavelets. It can be taken care of in our case much in the same way as in other settings (for instance by using the undecimated wavelet transform).

\section{Multifractal spectrum estimation}

Suppose that we want to estimate the spectrum $d(\alpha)$ of the signal $f$. The WSA representation yields a semi-parametric method through the following procedure. First, we segment $f$ into homogeneous parts using the algorithm described in the previous section. Each part $P_{i}, i=1, \ldots, p$ is well represented by a single WSA function $F_{i}$. Since we know the parameters of $F_{i}$, we can derive analytically its spectrum $d_{i}$ using Theorem 1 . Because there are a finite number of segments, the dimension $d(\alpha)$ associated to the Hölder exponent $\alpha$ for the whole signal will be the maximum of $d_{i}(\alpha), i=1, \ldots, p$. Thus, the semi-parametric estimation of $d(\alpha)$ using the WSA representation is:

$$
\hat{d}(\alpha)=\max _{i=1, \ldots, p} d_{i}(\alpha) .
$$

Of course, while each $d_{i}$ is concave, this will not be the case in general for $\hat{d}$. For instance, if $f$ is the lumping of two IFS, the estimated spectrum $\hat{d}$ will match the theoretical one and will exhibit the two bumps characteristic of the phase transition.

It is important to note that, for a given $\delta>0$, it is easy to construct two functions $f_{1}$ and $f_{2}$ such that $\left\|f_{1}-f_{2}\right\|_{L^{2}}<\delta$ or $\left\|f_{1}-f_{2}\right\|_{\infty}<\delta$, but the multifractal spectra of $f_{1}$ and $f_{2}$ are vastly different. Therefore, one can not in general conclude that the multifractal spectrum of the original signal will be close to the one of the approximating WSA signal. However, if one has some criterion which allows to check whether the approximating WSA signal has physical properties which are close to the ones of the original signal, then such a conclusion makes sense. For instance, in the case of speech signals that we consider below, an obvious criterion is just auditive comparison. In the application concerning Internet traffic, our criterion will be the comparison between our estimated spectrum and the one estimated using another approach. Indeed, since the two approaches are qualitatively different and give almost the same spectrum (as we will see), then it is likely that this spectrum is a good estimation of the true one.

\section{Numerical experiments}

Our first example is the representation of the word "welcome" uttered by a male speaker. The signal contains $2^{15}$ samples, we set $j_{0}=8$ and used the Daubechies 16 wavelet (as explained above, the choice of the wavelet is based on the two constraints (C1) and 
(C2)). In this application, as well as the next one, we set $\sigma(j)=j^{2}$. Using a threshold $\epsilon=50$ yields a modeling with seven WSA functions, where $64 \%$ of the coefficients are processed (the remaining $36 \%$ either correspond to levels in the tree above $j_{0}$ or have values greater than 1). As can be seen on figure 1, the original signal and the model are visually almost indistinguishable. More importantly, they sound practically the same, as the interested reader may check by pointing to http://www-rocq.inria.fr/fractales. In addition, the segmentation (see the vertical red lines) is phonetically relevant since the marks almost perfectly coincide with the following sounds : silence, /w/, / $\epsilon \mathrm{l} /$, silence, $/ \mathrm{k} /, / \mathrm{om} /$, silence. The slight discrepancy between the position of the segmentation marks and the exact location of the phonetic units is due to the fact that, in the current implementation, the marks are restricted to be on dyadic points.

In our second example, we present an application related to Internet traffic. We use a 512 samples log from the outgoing traffic at Berkeley, measured in bytes per time. The analyzing wavelet is Daubechies $4, j_{0}=4$ and $65 \%$ of the coefficients are processed. With a threshold of 30 we get 2 segments. Figure 2 displays the original log (in blue), its WSA approximation (in green), the segmentation marks (vertical red lines) and the estimated spectrum for each segment (right plot) obtained as explained in section 5. It is interesting to compare these spectra with the ones estimated in $[3]^{1}$ : the results are very similar, while if one uses more segments (or only one segment), there is a clear discrepancy (see figure 3 for the results obtained with four segments). Since the methods used here and in [3] are very different, such a match could be an indication that this particular log is indeed made of two parts, each of which is multifractally stationary. Such an information is useful for a better understanding of the traffic structure.

\section{Conclusion}

We have introduced in this work a new model for irregular signals. This model is versatile enough to handle a wide variety of practical situations, yet has enough structure to allow analytical computation of most fractal quantities of interest for processing. We have demonstrated on two examples how this approach permits to synthesize, segment and investigate the multifractal properties of signals. Further work will concentrate on enhancing the segmentation procedure and on applying this new tool to various types of signals (e.g. financial records).

\section{Appendix}

\section{Proof of Proposition 1}

Consider first the case where $n=j_{0}+1$. We seek $\lambda_{0}^{j_{0}+1}$ and $\lambda_{1}^{j_{0}+1}$ which realize

$\underset{1}{\operatorname{argmin}} \sum_{0 \leq k<2^{j_{0}+1}}\left|w_{k}^{j 0+1}-\tilde{w}_{k}^{j 0+1}\right|^{2}$.

$\begin{array}{clll}{ }^{1} \text { This paper can be down-loaded } & \text { from } & \text { www-rocq.inria.fr/fractales } & \text { or } \\ \text { www.ece.rice.edu/riedi/cv_publications.html } & \end{array}$


But $w_{k}^{j_{0}+1}=\operatorname{sgn}\left(w_{k}^{j_{0}+1}\right)\left|w_{\left[\frac{k}{2}\right]}^{j_{0}}\right| c_{k}^{j_{0}+1}$ and $\tilde{w}_{k}^{j_{0}+1}=\operatorname{sgn}\left(w_{k}^{j_{0}+1}\right)\left|w_{\left[\frac{k}{2}\right]}^{j_{0}}\right| \tilde{c}_{k}^{j_{0}+1}$ where $\tilde{c}_{k}^{j_{0}+1}$ is given by (3). Therefore, the problem is to solve

$$
\operatorname{argmin} \sum_{\substack{0 \leq k<2^{j_{0}+1} \\\left|c_{k}^{j_{0}+1}\right|<1}}\left|w_{\left[\frac{k}{2}\right]}^{j_{0}}\right|^{2}\left|c_{k}^{j_{0}+1}-\lambda_{r(k)}^{j_{0}+1}\right|^{2}
$$

where $r(k)$ equals 0 if $k$ is even and 1 if $k$ is odd. By splitting the sum over even and odd $\mathrm{k}$, this is equivalent to solving for $i=0,1$

$$
\operatorname{argmin} \sum_{\substack{0 \leq k<2^{j_{0}} \\\left|j_{2 k+i}^{j_{0}+1}\right|<1}}\left|w_{k}^{j_{0}}\right|^{2}\left|c_{2 k+i}^{j_{0}+1}-\lambda_{i}^{j_{0}+1}\right|^{2}
$$

After differentiating over $\lambda_{i}^{j_{0}+1}$ and setting the derivative to 0 , we get (4).

Suppose now that $n>j_{0}+1$ and that we have computed all the $\left(\lambda_{i}^{j}\right)$ for $j=j 0+$ $1, \ldots, n-1$. We then seek $\lambda_{0}^{n}$ and $\lambda_{1}^{n}$ which realize

$$
\operatorname{argmin} \sum_{0 \leq k<2^{n}}\left|w_{\left[\frac{k}{2^{n-j_{0}}}\right]}^{j_{0}}\right|^{2}\left|\prod_{j=j_{0}+1}^{n} c_{\left[\frac{k}{2^{n-j}}\right]}^{j}-\prod_{j=j_{0}+1}^{n} \tilde{c}_{\left[\frac{k}{2^{n-j}}\right]}^{j}\right|^{2} .
$$

Using the same arguments as in the case $n=j_{0}+1$, this is equivalent to solving for $i=0,1$

$$
\operatorname{argmin} \sum_{\substack{0 \leq k<2^{n-1} \\\left|c_{2 k+i}^{n}\right|<1}}\left|w_{\left[\frac{2 k+i}{2^{n-j} 0}\right]}^{j_{0}}\right|^{2}\left|c_{2 k+i}^{n} \prod_{j=j_{0}+1}^{n-1} c_{\left[\frac{2 k+i}{2^{n-j}}\right]}^{j}-\lambda_{i}^{n} \prod_{j=j_{0}+1}^{n-1} \tilde{c}_{\left[\frac{2 k+i}{2^{n-j}}\right]}^{j}\right|^{2} .
$$

After differentiating over $\lambda_{i}^{j_{0}+1}$ and setting the derivative to 0 , we get (5).

\section{References}

[1] M.F. Barnsley. Fractals Everywhere. AK Peters, 1993.

[2] K. Daoudi and J. Lévy Véhel and Y. Meyer. Construction of Continuous Functions with Prescribed Local Regularity. Constructive Approximation, (14):349-385, 1998.

[3] J. Lévy Véhel and R. Riedi. Fractional Brownian motion and data traffic modeling: The other end of the spectrum. Fractals in Engineering, Eds. J. Lévy Véhel, E. Lutton, C. Tricot, Springer Verlag, 1997.

[4] W. Leland, M. Taqqu, W. Willinger, and D. Wilson. On the Self-Similar Nature of Ethernet Traffic. IEEE Trans. on Networking, pages 1-15, Feb. 1994.

[5] S. Jaffard. Multifractal Formalism Functions, Part 1 and 2. SIAM J. Math. Anal., (28-4):944-970, 1997.

[6] K. Daoudi. Généralisations des IFS: Applications au traitement du signal. Ph.D. university Paris 9, 1996. http://www.loria.fr/equipes/parole. 


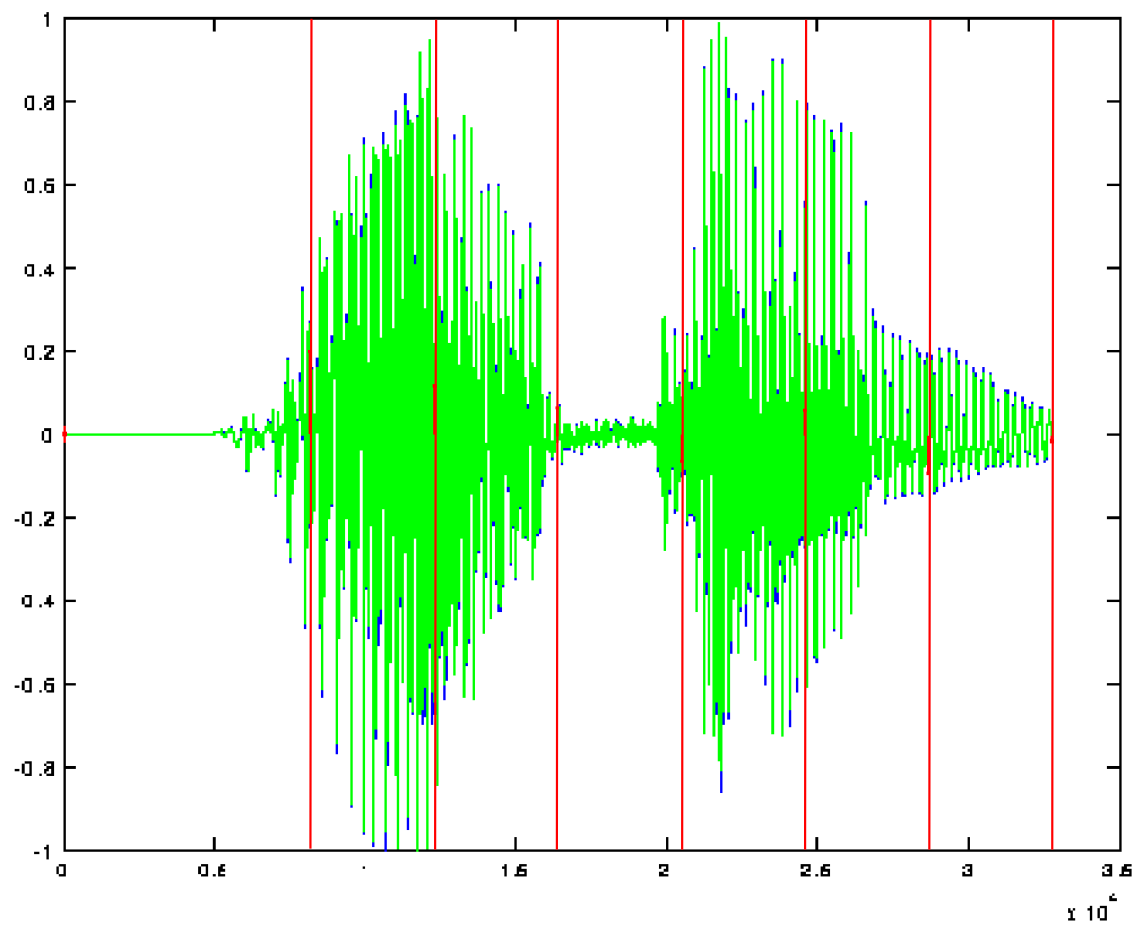

Figure 1: The word "welcome" uttered by a male speaker (in blue) along with its approximation (superimposed in green) and the segmentation marks (vertical red lines). 

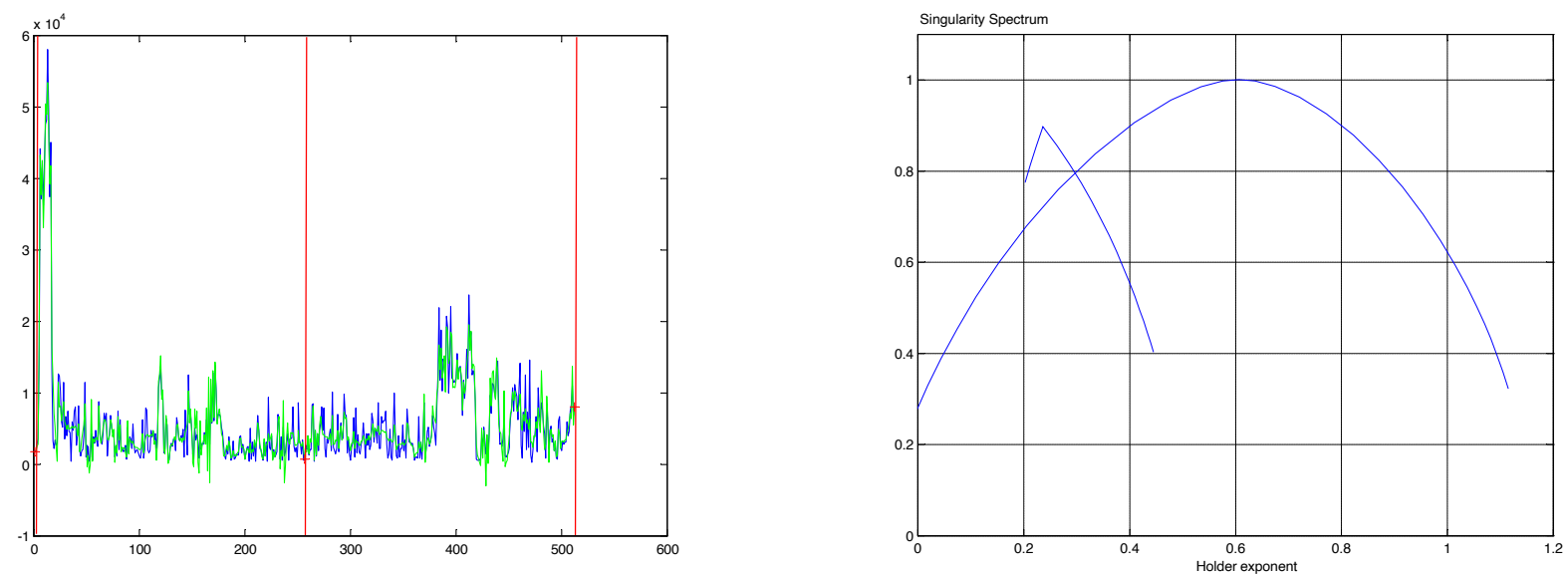

Figure 2: Left: Original log of Internet traffic (in blue), its WSA approximation (in green) using 2 segments and the segmentation marks (vertical red lines). Right: estimated spectrum for each segment. The estimated spectrum of the whole log is the upper envelope of the curves corresponding to each segment.
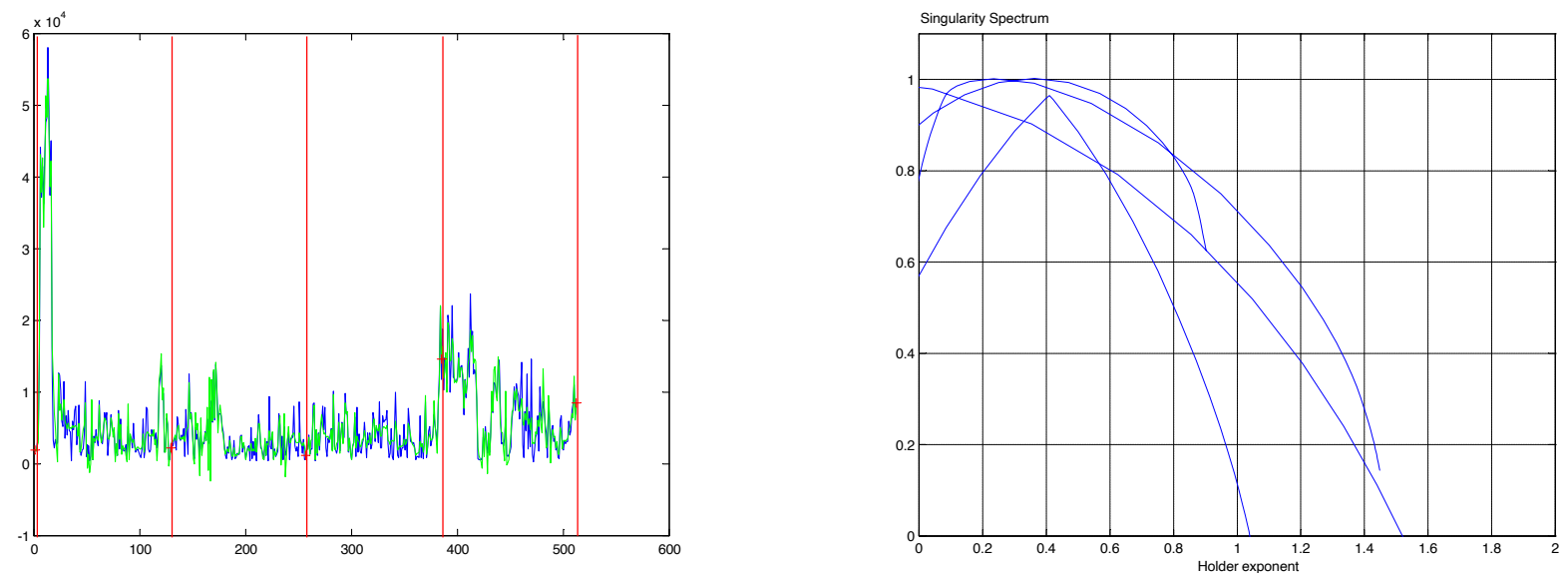

Figure 3: Left: Same Internet traffic log (in blue), its WSA approximation (in green) using 4 segments and the segmentation marks (vertical red lines). Right: estimated spectrum for each segment. The estimated spectrum of the whole log is the upper envelope of the curves corresponding to each segment. 